\title{
Theoretical Analysis of Effects of $\pi$-Conjugating Substituents on Building Blocks for Conducting Polymers
}

\author{
Ulrike Salzner* \\ Department of Chemistry, Bilkent University, 06533 Bilkent, Ankara, Turkey \\ J. B. Lagowski \\ Department of Physics and Physical Oceanography, Memorial University of Newfoundland, \\ St. J ohn's NF AlB 3X7, Canada \\ P. G. Pickup and R. A. Poirier \\ Department of Chemistry, Memorial University of Newfoundland, St. J ohn's, NF A1B 3X7, Canada
}

Received April 29, 1999

\begin{abstract}
Geometries of 4-dicyanomethylene-4H-cyclopenta[2,1-b:3,4-b'] dithiophene $\mathbf{1}$ and its $\mathrm{C}=\mathrm{O}, \mathrm{C}=\mathrm{S}$, $\mathrm{C}=\mathrm{CH}_{2}, \mathrm{C}=\mathrm{CF}_{2}$, and $\mathrm{C}=\mathrm{C}(\mathrm{SR})_{2}$ analogues were optimized using density functional theory. Three of the above groups, $\mathrm{C}=\mathrm{C}(\mathrm{CN})_{2}, \mathrm{C}=\mathrm{O}$, and $\mathrm{C}=\mathrm{S}$, were also examined on dipyrrole, difuran, dicyclopentadiene, and diborole. Electronic structures were analyzed with respect to their suitability as building blocks for conducting polymers with the natural bond orbital (NBO) method. All bridging groups investigated decrease HOMO-LUMO gaps compared to the unsubstituted parent dimers. Substitution affects HOMO and LUMO energies. Energy gap reduction is caused by a stronger decrease of LUMO energies compared to HOMO energies. The $\mathrm{C}=\mathrm{S}$ group leads to even smaller energy gaps than the dicyanomethylene group since the HOMO is lowered less in energy with $\mathrm{C}=\mathrm{S}$. Compared to unsubstituted dimers, the strongest substituent effects are found with pyrroles and furans. Boroles and thiophenes are least affected. The smallest HOMO-LUMO gaps are obtained for electron-poor systems such as boroles followed by cyclopentadienes. This is analogous to the trend for the unsubstituted parent systems. All of the bridging groups are potential $\pi$-acceptors due to their low-lying $\pi^{*}$-orbitals, and the corresponding polymers are predicted to be $\mathrm{n}$-dopable. In aromatic structures, the LUMO is localized around the bridging substituent and the coefficients at the $\alpha$-carbon atoms that reflect electron density are small. This might contribute to the poor conductivity of the n-doped form of poly-1. Electron-poor monomers and polymers tend to switch to quinoid structures. In quinoid repeat units, the HOMO is localized but not as strongly as the LUMO in the aromatic repeat units. The LUMO in quinoid repeat units is delocal ized with large coefficients at the $\alpha$-carbon atoms. Quinoid polymers could therefore be good conductors in the n-doped state.
\end{abstract}

\section{Introduction}

The discovery in 1973 that polysulfurnitride is a metal ${ }^{1}$ spurred enormous research effort and led to the creation of an entirely new class of chemical compounds, now known as conducting polymers. ${ }^{2}$ Conducting polymers have found applications not only due to their electrical properties but also due to their interesting optical properties. However, to this date, polysulfurnitride remains the one and only "synthetic metal" with no band gap. Most systems under investigation ${ }^{3}$ are semiconductors with band gaps ranging from 2 to $4 \mathrm{eV}$. These polymers are selected for technical applications because of their chemical stability and mechanical properties. Several polymers with band gaps below $1 \mathrm{eV}$ have been synthesized, ${ }^{4-6}$ and a copolymer with a band gap of less

* To whom correspondence should be addressed. Tel: (312) 2902122. Fax: (312) 266-4579. E-mail: salzner@fen.bilkent.edu.tr..

(1) André, J .-A.; Delhalle, J .; Brédas, J .-L. Quantum Chemistry Aided Design of Organic Polymers. An Introduction to the Quantum Chemistry of Polymers and its Applications; World Scientific: London, 1991.

(2) Skotheim, T. A. Handbook of Conducting Polymers; Marcel Dekker: New York, 1986

(3) Roncali, J. Chem. Rev. 1997, 97, 173-205.

(4) Ferraris, J. P.; Lambert, T. L. J. . Chem. Soc., Chem. Commun. 1991, 1268 than $0.16 \mathrm{eV}$ has been reported recently. ${ }^{6}$ However, none of the low band gap materials showed large intrinsic conductivity. The challenge to design and synthesize a stable synthetic metal therefore remains.

One strategy for decreasing the band gap is to bridge two thiophene rings with the dicyanomethylene group (Chart $1, \mathbf{1}) .^{4}$ The corresponding polymer has a band gap of $0.8 \mathrm{eV}$. This success was rationalized in terms of the electron-withdrawing nature of dicyanomethylene combined with symmetry considerations. Since the HOMO of dithiophene has a nodal plane through the center of the molecule, a substituent in the bridging position has little influence on the HOMO level. By electron withdrawal, the dicyanomethylene group thus lowers the energy of the LUMO with respect to the HOMO. Due to its low-lying LUMO, poly-1 has the additional advantage of being n-dopable. However, its conductivity in the n-doped form is low. The keto anal ogue of poly-1 has also been made and has a band gap of $1.2 \mathrm{eV} .^{7}$

(5) Havinga, E. E.; ten Hoeve, W.; Wynberg, H. Synth. Met. 1993, 55-57, 299-306.

(6) Huang, H.; Pickup, P. G. Chem. Mater. 1996, 8, 2212-2216.

(7) Lambert, T. L.; Ferraris, J . P. J . Chem. Soc., Chem. Commun 1991, 752-754. 
Chart 1<smiles></smiles><smiles>C=C1c2ccsc2-c2sccc21</smiles><smiles></smiles>
10<smiles>N#CC(C#N)=C1c2ccoc2-c2occc21</smiles><smiles>N#CC(C#N)=C1C2=C(BC=C2)C2=C1C=CB2</smiles>

16<smiles>FC(F)=C1c2ccsc2-c2sccc21</smiles><smiles>O=C1C2=C(CC=C2)C2=C1C=CC2</smiles><smiles>O=C1c2ccoc2-c2occc21</smiles><smiles>O=C1c2ccsc2-c2sccc21</smiles><smiles>S=C1c2ccsc2-c2sccc21</smiles>

3<smiles>C1=CSC(=C2c3ccsc3-c3sccc32)S1</smiles><smiles>S=C1C2=C(CC=C2)C2=C1C=CC2</smiles><smiles>S=C1c2cc[nH]c2-c2[nH]ccc21</smiles>

12<smiles>S=C1c2ccoc2-c2occc21</smiles><smiles>S=C1C2=C(BC=C2)C2=C1C=CB2</smiles>

18

The small band gap of poly-1 was also discussed in terms of alternating donor-acceptor moieties. ${ }^{8,9}$ According to this interpretation, the small band gap is due to the different positions of HOMO and LUMO in donors and acceptors. The final band gap would then result as the energy difference between the high-lying $\mathrm{HOMO}$ of the donor and the low-lying LUMO of the acceptor. ${ }^{5}$ The strongest effect was predicted for systems with the largest electronegativity differences between donor and acceptor. This donor-acceptor concept was suggested to work for organic polymers, in analogy to inorganic semiconductor crystals.

Polymer properties devel op gradually from molecular properties as the number of repeat units increases. The final band gap of a polymer is strongly influenced by two

(8) Bakhshi, A. K.; Yamaguchi, Y.; Ago, H.; Yamabe, T. Synth. Met. 1996, 79, 115-120.

(9) Toussiant, J . M.; Brédas, J . L. Synth. Met. 1995, 69, 637-640.

Chart 2

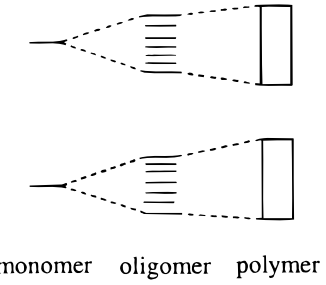

factors, the HOMO-LUMO gap of the repeat unit and the overlap between the repeat units, which determines the degree of level splitting and therefore the bandwidth (see Chart 2). To design a small band gap polymer, repeat units with small HOMO-LUMO gaps should be chosen and the overlap between the repeat units should be large. Large overlap is achieved with planar systems having large HOMO and LUMO coefficients at the sites where the repeat units are joined. Energy gaps and orbital coefficients can be determined for repeat units using calculations on finite systems. Toward this end, we are examining the suitability of compounds $\mathbf{1 - 1 8}$ shown in Chart 1 as building blocks for conducting polymers. A complication arises here, since some of the repeat units might be quinoid rather than aromatic in polymers. Hong and Song ${ }^{10}$ recently examined a series of polymers of this type (including poly-2, poly-3, poly-4, and poly-7) and found that polymers with electron donors in the outer rings have aromatic ground states. Therefore, 1-6 and 10-15 can be expected to form polymers with aromatic ground states. Compound $\mathbf{1 8}$ is already quinoid as a monomer. Compounds 7, 8, 16, and $\mathbf{1 7}$ are likely to undergo structural changes upon polymerization. These are included for comparison.

Investigating molecules al lows us to use higher levels of theory than is possible for polymers and to employ tools for analyzing wave functions that are unavailable for polymers. The aim of this study is to investigate the possibility of individually shifting levels if symmetry is exploited, since we found that substituents in the 3- and 4-positions of dithiophene shift HOMO and LU MO levels similarly and do not influence band gaps very much. ${ }^{11,12}$ We further study the origin of energy gap reductions in terms of electron-withdrawing effects versus the effect of $\pi$-conjugation. Comparison of cyclopentadiene, pyrrole, thiophene, and borole dimers allows us to analyze the effect of alternating donor-acceptor moieties. Promising repeat units for low band gap polymers are selected for explicit investigation of polymer properties.

\section{Methods}

Compounds $\mathbf{1}$ through $\mathbf{1 8}$ were optimized in $\mathrm{C}_{2 v}$ symmetry using density functional theory (DFT). Becke's three-parameter hybrid functional ${ }^{13}$ was combined with Perdew and Wang's correlation functional. ${ }^{14}$ Stevens-Basch-Krauss pseudopotentials ${ }^{15}$ and split valence plus polarization basis sets were empl oyed. ${ }^{16}$

(10) Hong, S. Y.; Song, J . M. J . Chem. Phys. 1997, 107, 1060710615.

(11) Salzner, U.; Kızıltepe, T. J Org. Chem. 1999, 64, 764-769.

(12) Salzner, U. Synth. Met., 1999, 101, 482-483.

(13) Becke, A. D. Phys. Rev. A 1988, 38, 3098-3100.

(14) Perdew, J . P. Phys. Rev. B 1986, 33, 8822-8824.

(15) Stevens, W.; Basch, H.; Krauss, J . J . Chem. Phys. 1984, 81 6026.

(16) Frisch, M. J .; Frisch, ÆE.; Foresman, J . B. Gaussian 94 User's Reference; Gaussian, Inc: : Pittsburgh, 1994-1995. 
The use of DFT orbital energies for estimating ionization potentials, electron affinities, and energy gaps is a subject of many studies ${ }^{17-26}$ and is beyond the scope of the present investigation. However, Savin et al. ${ }^{26}$ have shown that the exact Kohn-Sham orbitals provide excellent excitation energies. This agrees with our previous finding ${ }^{27-29}$ that hybrid functionals, which are generally superior to the local spin density approximation (LSDA) for chemical applications, greatly improve DFT band gaps over those obtained within LSDA. With a weight of the $30 \%$ of Hartree-Fock exchange, hybrid functionals yield HOMO-LUMO gaps in close agreement with $\lambda_{\max }$ values from UV spectroscopy. This functional is designated as B3P86-30\% and used in this investigation throughout. We found that IPs and EAs are about $1 \mathrm{eV}$ too low for oligomeric $\pi$-systems but that trends are reproduced correctly.

Substituent effects were analyzed in terms of localized orbitals with the natural bond orbital (NBO) method, which is based on natural population analysis (NPA). ${ }^{30-32}$ The essential feature of NBO analysis is that the el ectron density is represented, as far as possible, by localized core orbitals, bonds, and Ione pairs. These orbitals represent the molecules as localized Lewis structures. H owever, for conjugated systems Lewis structures are obviously not adequate. Deviations from ideal ized Lewis structure due to conjugation show up in NBO analysis as donor-acceptor orbital interactions of localized bonds and lone pairs with antibonds $\left(\pi \rightarrow \pi^{*}\right.$ and Ip $\left.\rightarrow \pi^{*}\right)$. The more delocalized the structure is, the lower are the occupancies of the bonds and Ione pairs and the larger are the occupancies of the antibonds. Thus, NBO analysis can be used to examine conjugation effects in $\pi$-conjugated systems quantitatively. All calculations were performed with Gaussian $94 .^{33}$

\section{Results}

In Table 1, negative HOMO energies, negative LUMO energies, and HOMO-LUMO gaps of bridged dimers are compared to those of the unsubstituted parent dimers. Calculations for unsubstituted dimers are based on trans conformations since these are lower in energy than the cis forms. The HOMO and LUMO energy levels of cis and trans forms are, nonetheless, practically identical. The

(17) Baerends, E. J .; Gritsenko, O. V. J . Phys. Chem. A 1997, 101, 5383-5403.

(18) Fritsche, L. Physica B 1991, 172, 7-17.

(19) Godby, R. W.; Schlüter, M.; Sham, L. J . Phys. Rev. B 1988, 37, 10159-10175.

(20) Levy, M. Phys. Rev. A 1995, 52, 50-52.

(21) Perdew, I. P.: Levy, M. Phys. Rev. Lett. 1983, 51, 1884

(22) Williams, A. R.; von Barth, U. In Theory of the Inhomogeneous Electron Gas; Lundqvist, S., March, N. H., Eds.; Plenum Press: London, 1983.

(23) Rösch, N.; Trickey, S. B. J . Chem. Phys. 1997, 106, 8940-8941.

(24) Chan, G. E.-L.; Tozer, D. J .; Handy, N. C. J . Chem. Phys. 1997, 107, 1536.

(25) Gal braith, J . M.; Schaefer, H. F. III. J . Chem. Phys. 1996, 105, $862-863$.

(26) Savin, A.; Umrigar, C. J .; Gonze, X. Chem. Phys. Lett. 1998, 288, 391-395.

(27) Salzner, U.; Lagowski, J . B.; Pickup, P. G.; Poirier, R. A. J Comput. Chem. 1997, 18, 1943-1953.

(28) Salzner, U.; Lagowski, J . B.; Pickup, P. G.; Poirier, R. A. J . Phys Chem. A 1998, 102, 2572-2578.

(29) Salzner, U.; Lagowski, J . B.; Pickup, P. G.; Poirier, R. A. Synth. Met. 1998, 96, 177-189.

(30) Reed, A. E.; Weinstock, R. B.; Weinhold, F. J . Chem. Phys. 1985 83, 735 .

(31) Reed, A. E.; Weinhold, F. J . Chem. Phys. 1985, 83, 1736. 899.

(32) Reed, A. E.; Curtiss, L. A.; Weinhold, F. Chem. Rev. 1988, 88,

(33) Gaussian 94: Frisch, M.J .; Trucks, G. W.; Schlegel, H. B.; Gill, P. M. W.; J ohnson, B. G.; Robb, M. A.; Cheeseman, J . R.; Keith, T.; Petersson, G. A.; Montgomery, J . A.; Raghavachari, K.; Al-Laham, M. A.; Zakrzewski, V. G.; Ortiz, J . V.; Foresman, J . B.; Peng, C. Y.; Ayala, P. Y.; Chen, W.; Wong, M. W.; Andres, J . L.; Replogle, E. S.; Gomperts, R.; Martin, R. L.; Fox, D. J .; Binkley, J . S.; Defrees, D. J .; Baker, J .; Stewart, J. P.; Head-Gordon, M.; Gonzalez, C.; Pople, J . A. Gaussian, Inc., Pittsburgh, PA, 1995.
Table 1. Negative HOMO Energies, Negative LUMO Energies, and HOMO-LUMO Gaps in eV for Compounds 1-18

\begin{tabular}{lccclccc}
\hline & HOMO & LUMO & $\mathrm{E}_{\mathrm{g}}$ & & HOMO & LUMO & $\mathrm{E}_{\mathrm{g}}$ \\
\hline dithio & 6.59 & 1.78 & 4.80 & dipyr & 5.82 & 0.33 & 5.49 \\
$\mathbf{1}$ & 7.21 & 3.98 & 3.23 & $\mathbf{1 0}$ & 6.46 & 3.16 & 3.29 \\
$\mathbf{2}$ & 6.88 & 3.02 & 3.86 & $\mathbf{1 1}$ & 6.02 & 1.95 & 4.06 \\
$\mathbf{3}$ & 6.83 & 3.64 & 3.19 & $\mathbf{1 2}$ & 6.02 & 2.77 & 3.24 \\
$\mathbf{4}$ & 6.40 & 2.25 & 4.16 & difur & 6.41 & 1.15 & 5.26 \\
$\mathbf{5}$ & 6.56 & 2.24 & 4.31 & $\mathbf{1 3}$ & 7.11 & 3.93 & 3.17 \\
$\mathbf{6}$ & 6.21 & 2.20 & 4.01 & $\mathbf{1 4}$ & 6.76 & 2.86 & 3.90 \\
dicp & 5.94 & 1.70 & 4.24 & $\mathbf{1 5}$ & 6.69 & 3.59 & 3.10 \\
$\mathbf{7}$ & 6.48 & 4.00 & 2.48 & dibor & 6.23 & 3.45 & 2.78 \\
$\mathbf{8}$ & 6.18 & 3.03 & 3.15 & $\mathbf{1 6}$ & 6.76 & 5.06 & 1.70 \\
$\mathbf{9}$ & 6.09 & 3.66 & 2.43 & $\mathbf{1 7}$ & 6.56 & 4.45 & 2.11 \\
& & & & $\mathbf{1 8}$ & 6.23 & 5.03 & 1.21
\end{tabular}

HOMO energy of trans dithiophene, for instance, is 6.59 $\mathrm{eV}$, and that of the cis form is $6.57 \mathrm{eV}$. The LUMO energies of both conformers are $1.78 \mathrm{eV}$. All bridging groups studied substantially lower energy gaps. For $\mathrm{C}=$ $\mathrm{S}$ lowerings between 2.25 and $1.57 \mathrm{eV}$, for $\mathrm{C}=(\mathrm{CN})_{2}$ between 2.2 and $1.08 \mathrm{eV}$, and for $\mathrm{C}=\mathrm{O}$ between 1.43 and $0.67 \mathrm{eV}$ result. The $\mathrm{C}=\mathrm{S}$ group is thus slightly more effective than the dicyanomethylene group. The effects with $\mathrm{C}=\mathrm{CH}_{2}, \mathrm{C}=\mathrm{CF}_{2}$, and $\mathrm{C}=\mathrm{C}(\mathrm{SCH})_{2}$ are smaller but nonnegligible.

With $\mathrm{C}=\mathrm{C}(\mathrm{CN})_{2}, \mathrm{C}=\mathrm{O}$, and $\mathrm{C}=\mathrm{S}$ bridging groups, the energy gap lowerings are larger for dipyrrole than for dithiophene. This leads to almost identical HOMOLUMO gaps for $\mathbf{1}$ and $\mathbf{1 0}$ and for $\mathbf{3}$ and $\mathbf{1 2}$, while the HOMO-LUMO gaps of dipyrrole and dithiophene differ by $0.69 \mathrm{eV}$. The range in all HOMO-LUMO gaps of the substituted dimers is $2 \mathrm{eV}$, a significant reduction compared to a range of $2.71 \mathrm{eV}$ for the parent dimers.

Although the HOMOs of these compounds have nodes at the position of the substituents, the HOMO energies are lowered with $\mathrm{C}=\mathrm{C}(\mathrm{CN})_{2}, \mathrm{C}=\mathrm{O}$, and $\mathrm{C}=\mathrm{S}$ in all but one case. The dicyanomethylene group has the strongest energy lowering effect, $0.53-0.7 \mathrm{eV}$. The $\mathrm{C}=\mathrm{S}$ group influences the HOMOs least and has no effect on the HOMO of 18. The HOMOs of furans are most strongly affected; cycl opentadienes and boroles show the smal lest effects. The differences are, however, rather small. With $\mathrm{C}=\mathrm{CH}_{2}, \mathrm{C}=\mathrm{CF}_{2}$, and $\mathrm{C}=\mathrm{C}(\mathrm{SCH})_{2}$ the $\mathrm{HOMO}$ energies increase slightly.

The LUMO energies of all the bridged dimers are substantially lower than those of the unsubstituted dimers. The dipyrrole LUMO energies are lowered more than the dithiophene LUMO energies upon bridging. Therefore, the difference between LUMO energies of bridged dimers of pyrrole and thiophene is much smaller than that between unsubstituted dipyrrole and dithiophene. The dicyanomethylene group is most efficient in lowering $\mathrm{LUMO}$ energies, followed by $\mathrm{C}=\mathrm{S}$ and $\mathrm{C}=\mathrm{O}$. The covalent $\mathrm{C}=\mathrm{CR}_{2}$ groups have smaller effects on LUMO energies. Electronegative fluorines as in $\mathrm{C}=\mathrm{CF}_{2}$ do not enhance the LUMO energy lowering.

In Table 2, $\pi$-electron densities in the four carbon double bonds and total $\pi$-charges on the eight carbon atoms of the backbone of compounds $\mathbf{1 - 1 8}$ are listed as obtained with NBO analysis; $\pi$-charges are decomposed into $\pi$ and $\pi^{*}$ contributions to examine the extent of conjugation. In Table 3, the same information is compiled for the bridging groups. The trend in $\pi$-charges of the carbon backbone reflects the trend in $\pi$-donor strengths of the heteroatoms. Strong donors such as NH, S, and O lead to negative carbon $\pi$-backbones. With $\mathrm{CH}_{2}$, the 
Table 2. Number of Electrons in Carbon $\pi$ and $\pi^{*}$ Orbitals, Total $\pi$-electron Densities in the C $=\mathrm{C} \pi$-system, Total $\pi$-charges, and Total Charges on the Carbon Backbone of 1-18

\begin{tabular}{lccccc}
\hline & $\pi$ & $\pi^{*}$ & $\pi_{\text {tot }}$ & $\pi_{\text {charge }}$ & total charge \\
\hline dithio & 7.36 & 1.30 & 8.66 & -0.66 & -2.08 \\
$\mathbf{1}$ & 7.24 & 1.42 & 8.66 & -0.66 & -1.72 \\
$\mathbf{2}$ & 7.28 & 1.36 & 8.64 & -0.64 & -1.86 \\
$\mathbf{3}$ & 7.24 & 1.38 & 8.62 & -0.62 & -1.76 \\
$\mathbf{4}$ & 7.30 & 1.42 & 8.72 & -0.72 & -1.76 \\
$\mathbf{5}$ & 7.28 & 1.44 & 8.72 & -0.72 & -1.76 \\
$\mathbf{6}$ & 7.26 & 1.50 & 8.76 & -0.76 & -1.78 \\
dicp & 7.44 & 0.64 & 8.08 & -0.08 & -1.22 \\
$\mathbf{7}$ & 7.34 & 0.76 & 8.10 & -0.10 & -0.82 \\
$\mathbf{8}$ & 7.38 & 0.64 & 8.02 & -0.02 & -0.96 \\
$\mathbf{9}$ & 7.36 & 0.70 & 8.06 & -0.06 & -0.86 \\
dipyr & 7.26 & 1.46 & 8.72 & -0.72 & -0.88 \\
$\mathbf{1 0}$ & 7.10 & 1.52 & 8.62 & -0.62 & -0.40 \\
$\mathbf{1 1}$ & 7.16 & 1.48 & 8.64 & -0.64 & -0.56 \\
$\mathbf{1 2}$ & 7.12 & 1.48 & 8.60 & -0.60 & -0.44 \\
difur & 7.34 & 1.14 & 8.48 & -0.48 & -0.22 \\
$\mathbf{1 3}$ & 7.22 & 1.22 & 8.44 & -0.44 & +0.20 \\
$\mathbf{1 4}$ & 7.26 & 1.18 & 8.44 & -0.44 & +0.06 \\
$\mathbf{1 5}$ & 7.24 & 1.22 & 8.46 & -0.46 & +0.18 \\
dibor & 7.32 & 0.40 & 7.72 & +0.28 & -2.26 \\
$\mathbf{1 6}$ & 7.38 & 0.42 & 7.80 & +0.20 & -1.92 \\
$\mathbf{1 7}$ & 7.42 & 0.30 & 7.72 & +0.28 & -1.56 \\
$\mathbf{1 8}$ & 6.68 & 0.50 & 7.18 & +0.82 & -1.26
\end{tabular}

Table 3. Number of Electrons in Bridging Group $\pi$ and $\pi^{*}$ Orbitals, Total $\pi$-electron Densities in the Bridging Groups, $\pi$-Charges, and Total Charges on the Bridging Groups of 1-18

\begin{tabular}{rccccc}
\hline & $\pi$ & $\pi^{*}$ & $\pi_{\text {tot }}$ & $\pi_{\text {charge }}$ & total charge \\
\hline $\mathbf{1}$ & 5.67 & 0.40 & 6.07 & -0.07 & -0.15 \\
$\mathbf{2}$ & 1.96 & 0.14 & 2.10 & -0.10 & 0.06 \\
$\mathbf{3}$ & 1.91 & 0.19 & 2.10 & -0.10 & -0.07 \\
$\mathbf{4}$ & 1.86 & 0.09 & 1.95 & +0.05 & 0.06 \\
$\mathbf{5}$ & 1.85 & 0.22 & 2.07 & -0.07 & 0.02 \\
$\mathbf{6}$ & 7.24 & 0.58 & 7.82 & +0.18 & 0.08 \\
$\mathbf{7}$ & 5.67 & 0.33 & 6.00 & 0.0 & -0.09 \\
$\mathbf{8}$ & 1.95 & 0.10 & 2.05 & -0.05 & -0.08 \\
$\mathbf{9}$ & 1.90 & 0.13 & 2.03 & -0.03 & -0.03 \\
$\mathbf{1 0}$ & 5.70 & 0.43 & 6.13 & -0.13 & -0.18 \\
$\mathbf{1 1}$ & 1.96 & 0.15 & 2.11 & -0.11 & 0.04 \\
$\mathbf{1 2}$ & 1.92 & 0.21 & 2.13 & -0.13 & -0.10 \\
$\mathbf{1 3}$ & 5.68 & 0.36 & 6.04 & -0.04 & -0.12 \\
$\mathbf{1 4}$ & 1.96 & 0.12 & 2.08 & -0.08 & 0.08 \\
$\mathbf{1 5}$ & 1.91 & 0.17 & 2.08 & -0.08 & -0.05 \\
$\mathbf{1 6}$ & 5.62 & 0.33 & 5.95 & +0.05 & -0.06 \\
$\mathbf{1 7}$ & 1.94 & 0.09 & 2.03 & -0.03 & 0.07 \\
$\mathbf{1 8}$ & 1.95 & 0.56 & 2.51 & -0.51 & -0.07 \\
$\mathbf{1 9}$ & 1.86 & 0.14 & 2.00 & 0.0 & 0.0
\end{tabular}

$\pi$-backbone is almost neutral and with $\mathrm{BH}$ it is positively charged. Adding bridging groups has relatively little influence on the total $\pi$-charges of the carbon backbone compared to the unsubstituted dimers. The $\pi$-charges on the bridging groups are small and sometimes even positive. Note that the dicyanomethylene group in electrondeficient borole $\mathbf{1 6}$ has a positive $\pi$-charge, and in cyclopentadiene 7 the dicyanomethylene group is neutral. Decomposition of $\pi$-electron densities reveals, however, that $\pi$-occupancies decrease and $\pi *$-occupancies increase upon bridging. Thus, bridging groups increase conjugation but do not withdraw $\pi$-electrons.

Total charges on the carbon atoms of the backbone (last column in Table 2 ) reflect the total, $\sigma$ plus $\pi$, effect of bridging groups and heteroatoms. With both $\mathrm{S}$ and $\mathrm{BH}$, the carbon backbone is negatively charged. With $\mathrm{S}$ this is due to the $\pi$-donor ability of $\mathrm{S}$. With $\mathrm{BH}$ it is due to the polarity of the $\mathrm{B}-\mathrm{C} \sigma$-bonds. Furans have positive carbon backbones due to the large electronegativity of

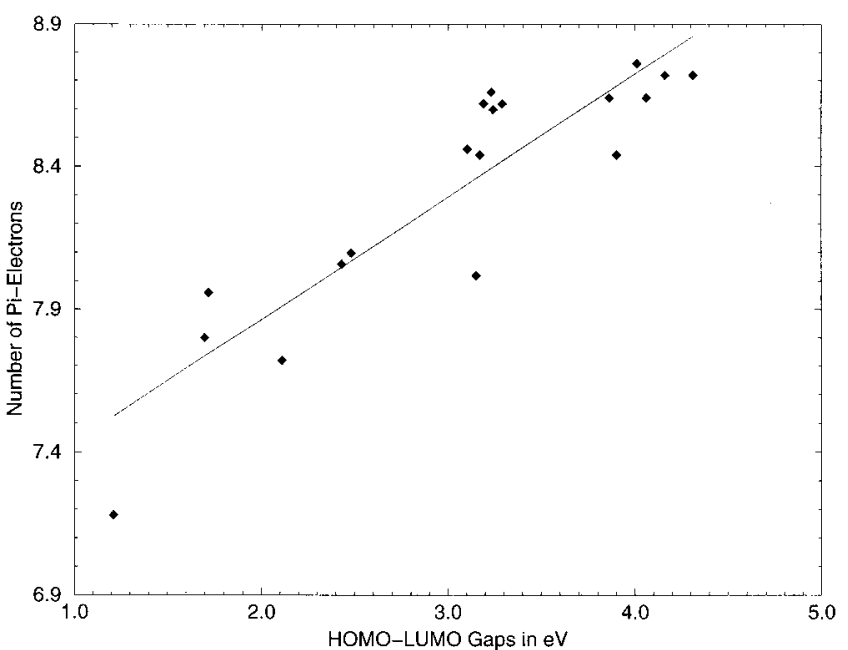

Figure 1. Correlation between number of $\pi$-electrons in the carbon backbone and energy gaps for compounds 1-18 and 19 (see Discussion). A neutral backbone would contain eight el ectrons.

oxygen. Pyrroles have negative total charges on the backbone since $\mathrm{N}$ is less electronegative than $\mathrm{O}$ and a better $\pi$-donor.

Comparison of the bridged dimers to the parent dimers indicates that the bridging groups decrease the total electron density on the carbon backbone by between 1.0 and 0.2 electrons. However, the small and sometimes positive charges on the bridging groups show that the bridging groups do not accept this electron density. For instance, upon bridging with $\mathrm{C}=\mathrm{O}$, the carbon atoms of the $\pi$-system lose about 0.3 electrons. But the charges on the $\mathrm{C}=\mathrm{O}$ groups range from +0.08 e to -0.08 electrons. Upon bridging with dicyanomethylene, the charges on the backbone carbon atoms drop by $0.4-0.6$ electrons. The charges on the dicyanomethylene group, however, range only from -0.09 to -0.15 electrons. Thus, carbon atoms of the backbone lose electrons by rearrangement of charge within the backbone. Most of the electron density goes to the hydrogens and to the heteroatoms rather than to the bridging groups.

Within the series of bridged dimers, a rough correlation between total $\pi$-densities and energy gaps seems to be present. In Figure 1, total $\pi$-densities of $\mathbf{1 - 1 8}$ are plotted against energy gaps. Although the correlation is far from perfect, the correlation coefficient of 0.89 shows that there is a connection between $\pi$-density and energy gap. No correlation was found between total charges on the backbone or total charges on the bridging groups and energy gaps.

Inclusion of the results for parent dimers into the data set lowers the correlation coefficients between $\pi$-density and energy gap. This is due to the fact that bridging groups decrease the energy gap by resonance rather than by charge withdrawal (inductive effect). In Figure 2, the stabilization energies associated with the donor-acceptor interactions from the carbon $\pi$-system to the bridging groups are plotted against the energy gap reductions compared to those for the parent dimers. The correlation coefficient of 0.90 indicates that the donor-acceptor interaction between the $\pi$-system of the backbone and the LUMO of the bridging group is the dominating influence for the energy gap reduction.

In Table 4, HOMO and LUMO orbital coefficients at the $\alpha$-carbon atoms are compiled for $\mathbf{1}, \mathbf{3}, \mathbf{1 0}, \mathbf{1 2}, \mathbf{1 6}$, and 


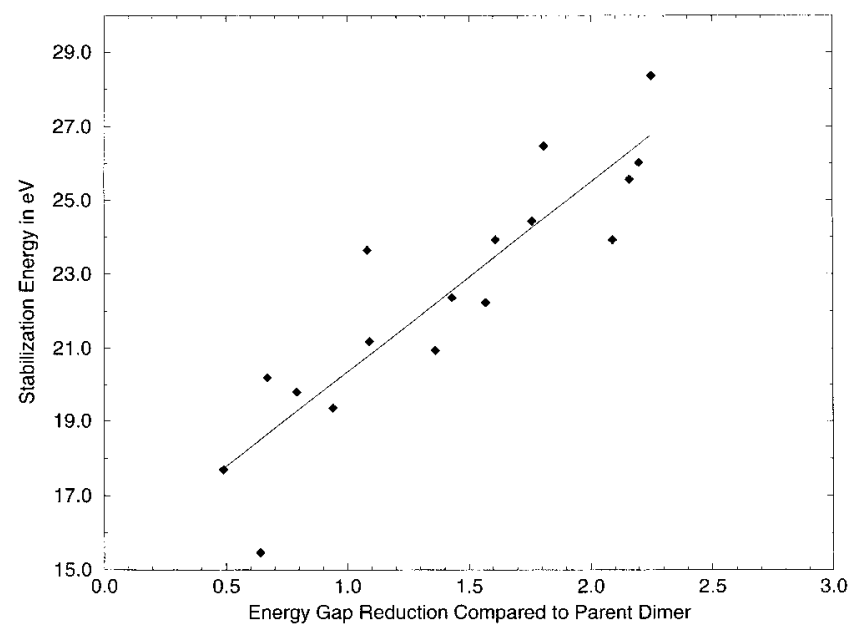

Figure 2. Correlation between the energy gain associated with the $\pi-\pi^{*}$ donor-acceptor interaction from the backbone to the bridging group and the energy gap reduction compared to the unsubstituted dimers for 1-17. Compound 18 is not included since it has a quinoid structure. For this reason, the $\pi$-bond that is involved in the donor-acceptor interaction used for this plot does not exist.

Table 4. Orbital Coefficients in HOMO and LUMO at the $\alpha$-Carbons of Compounds $1,3,10,12,16$, and 18

\begin{tabular}{rccccc}
\hline & HOMO & LUMO & & HOMO & LUMO \\
\hline $\mathbf{1}$ & 0.327 & 0.094 & $\mathbf{1 2}$ & 0.338 & 0.033 \\
& 0.185 & 0.065 & & 0.201 & 0.012 \\
$\mathbf{3}$ & 0.325 & 0.090 & $\mathbf{1 6}$ & 0.292 & 0.093 \\
& 0.189 & 0.058 & & 0.158 & 0.061 \\
$\mathbf{1 0}$ & 0.339 & 0.036 & $\mathbf{1 8}$ & 0.168 & 0.325 \\
& 0.196 & 0.019 & & 0.099 & 0.218
\end{tabular}

18. Since a split valence basis set was used, there are two coefficients for each site. The $\alpha$-carbon atoms have substantial coefficients in the HOMOs but very small contributions to the LUMOs. For quinoid 18, HOMO and LUMO are reversed compared to those of the aromatic compounds. Therefore, in 18, the HOMO has small coefficients at the $\alpha$-carbon atoms while the LUMO coefficients are large. However, the coefficients in the HOMO of $\mathbf{1 8}$ are about three times larger than those in the LUMOs of the aromatic compounds.

\section{Discussion}

The polymer of $\mathbf{1}$ is one of the lowest band gap organic polymers synthesized so far. The band gap was determined to be $0.8 \mathrm{eV}, 1.2 \mathrm{eV}$ less than that of polythiophene. Our calculations show that the energy gap of $\mathbf{1}$ is lowered by $1.57 \mathrm{eV}$ compared to that of dithiophene. The Iarger lowering compared to experimentally determined difference between the polymers indicates that the energy gap of $\mathbf{1}$ decreases slower than that of dithiophene with increasing chain length. The reason is most likely that the LUMO of $\mathbf{1}$ has very small coefficients at the $\alpha$-carbon atoms (Table 4) at which polymerization occurs. This leads to a flat conduction band and a slow decrease in the energy gap with increasing chain length. In other words, the low-lying LUMO of $\mathbf{1}$ is localized at the bridging group, preventing conduction band formation in the polymer. This reasoning is consistent with experimental results on a copolymer containing $\mathbf{1}$ that show that the LUMO energy does not decrease much upon polymerization. Charge carrier localization in the conduction band might explain the lower mobility of n-type charge carriers as compared to p-type carriers. Although conductivity is a bulk property and although dedoping might play a significant role, intrinsic limitation of electron mobility in the conduction band could be an alternative explanation for the observed lower conductivity of the n-doped form of the polymer compared to that of the $p$-doped form. ${ }^{6,34}$

The band gap reduction of poly-1 is usually attributed to the electron-withdrawing ability of the dicyanomethylene group. ${ }^{3}$ The absence of a correlation of energy gaps with total charges proves this argument wrong. As can be seen from Table 3 , even positively charged bridging groups $\left(\mathrm{C}=\mathrm{O}, \mathrm{C}=\mathrm{CH}_{2}, \mathrm{C}=\mathrm{CF}_{2}\right.$, and $\left.\mathrm{C}=\mathrm{C}(\mathrm{SR})_{2}\right)$ lower energy gaps significantly compared to those for the parent dimers; $\pi$-densities (rather than total charges) were shown in Figure 1 to correlate with energy gaps. The $\pi$-charges are, however, dominated by the $\pi$-donor strengths of the heteroatoms while bridging groups do not influence the $\pi$-charges very much. Examination of the $\pi$-charges (second last col umn in Table 3 ) shows that the dicyanomethylene group can be a $\pi$-donor, be neutral, or act as a $\pi$-acceptor, depending on the $\pi$-electron density in the backbone. Nonetheless, it lowers the energy gap in every case. Figure 2 shows that the energy gap reductions can be correlated with the strengths of the donor-acceptor interactions between the backbones and the bridging groups. Thus, extension of the $\pi$-system onto the bridging groups rather than electron withdrawal is the dominating effect for energy gap reductions with bridging groups. This explains why bridging groups with high and with lower electronegativity (e.g., compare 1 with $\mathbf{3}$ or $\mathbf{6}$ ) reduce energy gaps and why el ectronegative atoms do not enhance the gap reductions (e.g., compare $\mathbf{4}$ with $\mathbf{5}$ and $\mathbf{2}$ with $\mathbf{3}$ ).

According to Pauling, ${ }^{35}$ el ectronegativity is the power of an atom or group to attract shared el ectrons to itself. Since the charges on the bridging groups in 1-18 are small, none of these bridging groups qualifies as being strongly electronegative according to Pauling's definition. According to Mulliken, ${ }^{35}$ electronegativity can be estimated as the average between ionization potential (IP) and electronaffinity (EA), eq 1.

$$
E N=(I P+E A) / 2
$$

This definition leads to large ENs for all bridging groups considered because their low-lying $\pi^{*}$-orbitals are associated with large el ectronaffinities. Whether groups such as dicyanomethylene should be considered electronegative is thus a matter of which definition is preferred. Dicyanomethylene is, however, not a strongly electron-withdrawing substituent in the neutral ground state of these molecules. It rather is a potential electron acceptor with respect to $n$-doping or el ectronic excitation.

The low band gap of poly-1 was also discussed in terms of alternating donor-acceptor substitution. ${ }^{8,9}$ This interpretation is based on behavior observed with inorganic semiconductors. For this concept to apply for organic polymers, the band gap of the donor-acceptor polymer would have to arise approximately as the energy difference between the high-lying HOMO of the donor and the low-lying LUMO of the acceptor..$^{5}$ According to this argument, the energy gap of $\mathbf{1 0}$ involving the stronger

(34) Huang, H.; Pickup, P. G. Acta Polym. 1997, 48, 455-457.

(35) Christen, H. R. Grundlagen der allgemenen und angewandten Chemie; Otto Salle Verlag: Frankfurt, 1980. 
donor $\mathrm{NH}$ should be smaller than that of $\mathbf{1}$ since the HOMO of dipyrrole is higher in energy than the HOMO of dithiophene. This is not observed. In contrast, energy gaps with $\mathrm{CH}_{2}$ and the acceptor group $\mathrm{BH}$ are much smaller than those of the donor-acceptor systems. The reason for this is that HOMO and LUMO levels of the present donor-acceptor systems are fully conjugated. Since HOMO and LUMO levels of building blocks (aromatic and fulvenic rings) are not preserved in the del ocalized fused organic $\pi$-systems, the donor-acceptor concept does not work for the building blocks examined here. It is unlikely that this delocalization would disappear upon polymerization. Thus, donor-acceptor substitution is not the reason for the low band gaps of polymers containing these repeat units.

Compounds 7-9 and 16-18, which do not contain electron-donating heteroatoms, show smaller energy gaps than 1. Considering that the experimental band gap of poly-1 is $2.4 \mathrm{eV}$ smaller than our calculated energy gap of monomer 1, poly-7-9 and 16-18 with energy gaps for the monomers between 1.21 and $3.15 \mathrm{eV}$ might have very small band gaps if aromatic structures were preserved in the corresponding polymers. However, as shown by $\mathrm{H}$ ong and Song, ${ }^{10}$ polymers that lack electron donors in the outer rings are likely to adopt quinoid structures with larger band gaps than predicted for the aromatic forms. Nonetheless, even the quinoid polymers calculated by Hong and Song have band gaps in the range of only about $1 \mathrm{eV}$. The most el ectron-deficient compound we examined here, 18, is al ready quinoid as a monomer. Thus, switching to a quinoid structure cannot increase the energy gap of 18. It is interesting to note that for quinoid $\mathbf{1 8} \alpha$-carbon atoms have large coefficients in the LUMO (Table 4). If this translates to polymers, quinoid polymers might be better conductors in the $\mathrm{n}$-doped form than aromatic ones. Conductivity in the p-doped form of quinoid polymers might not be as poor as that of $n$-doped aromatic polymers since the orbital coefficients at the $\alpha$-carbon atoms are still significantly larger. Poly-18 is thus the most promising candidate for a conducting polymer.

Boroles are conceptually interesting, but it is questionable whether stable boroles can ever be realized. However, other systems that lack $\pi$-donors in the outer rings may behave in a similar way. The dicyanomethylene group might not be suitable since adjacent $\mathrm{C}=\mathrm{C}(\mathrm{CN})_{2}$ groups would lead to sterically crowded systems. The $C=$ $\mathrm{S}$ group therefore appears to be an alternative. For this reason, we performed calculations for $\mathbf{1 9}$, which has three $\mathrm{C}=\mathrm{S}$ groups. The energy gap of the monomer is $1.72 \mathrm{eV}$, similar to that of borole 16. The structure of monomer 19 is aromatic, but the dimer is quinoid with an energy gap of $0.51 \mathrm{eV}$ and the quinoid trimer has an energy gap of only $0.27 \mathrm{eV}$. Oligomers of $\mathbf{1 9}$ are presently being investigated by one of us (U.S.).

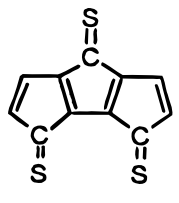

19

Bridging groups reduce energy gaps compared to those of the parent dimers but influence thiophenes, pyrroles, cyclopentadienes, furans, and boroles to different degrees. Electron-deficient systems such as boroles show the smallest response. Cyclopentadiene is second, followed by furan and pyrrole. Thiophenes show smaller effects than pyrroles and furans, although sulfur lone pairs are strong donors. Table 3 shows that in $\pi$-electron-rich compounds, the bridging groups tend to accumulate slightly more $\pi$-charge than in electron-poor ones. Comparison of the occupancies of the sulfur lone pairs in dithiophene and bridged dithiophenes (not given in Table 3) shows that the sulfur Ione pair occupancies decrease while $\pi$-charges in the carbon backbone stay almost constant when conjugating bridging groups are introduced. Thus, sulfur lone pairs partially compensate for substituent effects. The occupancies of the nitrogen lone pairs are unaltered in the bridged dimers compared to dipyrrole and the $\pi$-density in the backbone decreases upon introduction of bridging groups. This may explain the larger energy gap reduction with pyrrole than with thiophene.

\section{Conclusions}

Investigation of 18 bridged dimers of thiophene, cyclopentadiene, pyrrole, furan, and borole showed that two factors determine the size of the energy gaps. The first is the $\pi$-electron density in the carbon backbone and is correlated with the $\pi$-donating ability of the heteroatoms in the outer rings. To reduce energy gaps, $\pi$-electrondonating heteroatoms should be avoided. The second factor is the extension of the $\pi$-system onto the bridging groups. Bridging groups that contain $\pi$-systems themselves extend $\pi$-conjugation especially in the LUMO and lower energy gaps. Charges on bridging groups do not correlate with energy gaps, indicating that electron withdrawal is not the dominating factor. The energy gaps are largest when both the heteroatoms in the outer rings and the bridging groups are $\pi$-donors and smallest when both are $\pi$-acceptors. Alternating donor-acceptor substitution leads to intermediate energy gaps. There is thus no special energy gap reduction due to donor-acceptor substitution.

LUMO coefficients at the $\alpha$-carbon atoms of aromatic forms of 1-17 are very small. The LUMOs are localized at the bridging groups and the central ring. This explains why polymers of these systems have flat conduction bands. Thus, although these systems are good candidates for $\mathrm{n}$-doping due to their low LUMO energies, they might intrinsically be poor conductors in then-doped state. This would be an alternative explanation for the poor conductivity of the n-doped state of conducting polymers that is usually attributed to undoping and poor chemical stability. Since conductivity is a bulk property, gas-phase calculations cannot give a final answer to this problem. In quinoid systems, HOMO and LUMO are inverted as compared to the aromatic forms. Therefore, the LUMOs would be conjugated in the polymers, while the HOMOs might be localized. Thus, if our above argument turns out to be correct, quinoid polymers might be stable n-dopable systems with good conductivity in the n-doped state. Conductivity in the p-doped state might be relatively low.

The combination of electron-deficient five-membered rings such as boroles and thiofulvenes with $\pi$-conjugating bridging groups seems to generate promising building blocks for polymers with extremely small energy gaps. Polymers involving borole and thiofulvene are under investigation. These polymers will probably be quinoid. 
Effects of $\pi$-Conjugating Substituents

Intrinsic conductivity might be significant if poor conjugation in the valence band can be compensated for by good conjugation in the conduction band.

Acknowledgment. We thank the Natural Sciences and Engineering Research Council of Canada and
J . Org. Chem., Vol. 64, No. 20, 19997425

Memorial University for financial support. We also thank Bilkent University, the Computing and Communications Department of Memorial University, and Digital, I nc. for computing resources.

J O990725P 